\title{
Levels of dependency in local authority homes for the elderly
}

\author{
T. BOOTH, A. BARRITT, S. BERRY, D. N. MARTIN, C. MELOTTE, AND S. STONE \\ From the Joint Unit for Social Services Research, Department of Sociological Studies, University of Sheffield
}

In this article we report the results from an interim analysis of a census of residents in homes for the elderly in four local authority social services departments. The census was undertaken by the Joint Unit for Social Services Research at Sheffield University, which brings together academic research workers and senior research staff from local authority social services departments and allied agencies. ${ }^{1}$ It was designed to provide a broad picture of the characteristics of residents and the pattern of dependency.

Throughout the paper we have used the concept of dependency in preference to the notion of functional handicap in order to convey how the abilities and incapacities which residents present may be seen in part as a quality or consequence of their relationships with others-especially their carers-rather than simply as personal attributes rooted in the facts of illness, impairment, or old age.

\section{Method}

The officers in charge of all local authority homes for the elderly in each of the four social services departments taking part in the study were invited to complete a questionnaire ${ }^{12}$ on every person who occupied a bed in their homes, including those temporarily not resident for whom a bed was being reserved.

The questionnaire was a revised version of one originally used in an earlier survey in Sheffield, which served as a convenient and helpful pilot study for the present project. $^{2}$ Using the questionnaire, the officers were asked to describe each resident in terms of self-care ability (including mobility and continence), mental state, behaviour, and extent of social integration within the home. Additional information on sex, age, age on admission, and length of stay was also collected for each resident.

Rigorous efforts were made to ensure consistency between the officers in their interpretation and completion of the questionnaires. Briefing sessions were held to outline the aims of the study, to explain the mechanics of the exercise, and to clarify any uncertainties about the use of the questionnaire.

Two points in particular were emphasised during these sessions. Firstly, that the study was concerned with the functional skills of residents rather than with their physical disabilities or conditions. Secondly, that the questionnaire was intended to assess the personal functioning of residents within the home and not designed to measure how they might manage in other environments or with different medical treatment.

\section{Results}

\section{RESPONSE}

The census as undertaken early in 1980 . Questionnaires on 6947 residents were returned from the $\mathbf{1 7 5}$ local authority homes for the elderly in the four areas, making the stuidy the largest of its kind since the DHSS census of residential accommodation in 1970. Of the enumerated population, 6680 (96.2\%) were permanent residents and 267 (3.8\%) occupied short-stay places. Table 1 provides a breakdown of the response by area.

Table 1 Census response by local authority

\begin{tabular}{lcl}
\hline & No. of homes & No. of residents \\
\hline North Yorkshire & 45 & 1882 \\
Derbyshire & 51 & 2071 \\
Kirklees & 33 & 1182 \\
Nottinghamshire & 46 & 1812 \\
TOTAL & 175 & 6947 \\
\hline
\end{tabular}

The questionnaires were carefully edited and checked for internal consistency within each of the authorities so that ambiguities or omissions could be corrected and verified with the officers in charge of the homes. Furthermore, reliability checks on the data were made, using subsamples of the census population: 
(a) Test-retest checks were undertaken in two homes where the principals agreed to complete the same questionnaire on the same residents a fortnight after the initial census. There was a high correlation between the two sets of results, with $95 \%$ of responses on the second application matching those given on the first assessment.

(b) Interrater checks, in which different members of the residential care staff completed independently the same questionnaire on the same residents, were made in two homes using four different pairs of staff. Again, the results showed a high correlation between the assessments of residents: overall, $83 \%$ of the ratings of the first assessor were verified by the second member of staff.

The results of these reliability checks, which will be reported in detail in due course, give us confidence that the findings of the census represent an honest expression by residential staff of their perceptions of the functioning of residents in their homes. Indeed, it is worth emphasising that the data are derived from the personal knowledge of residents acquired by officers in charge and their staffs as a result of their close and lengthy acquaintance with the old people in their care $(85 \%$ of residents had been living in their homes for six months or more).

THE SCALES

As part of our analysis, the 6947 residents were classified individually on four separate scales, each designed to provide a composite rating of an aspect of personal functioning: a self-care scale, a continence scale, a social integration scale, and an orientation scale.

\section{Self-care scale}

This classified residents as severely dependent, moderately dependent, or independent, according to their mobility and their performance when washing, dressing, feeding, bathing, and using the WC.

(a) Residents were classified as severely dependent if they were chairbound and unable to get about without help, or if they were bedfast, or if they were unable to perform any three of the following tasks without considerable help from the staff: washing, dressing, feeding, bathing, and using the WC.

(b) Residents who were incontinent of urine on average ambulant and mobile, with or without aids but without personal assistance, and also fully capable of dressing, feeding, washing, and using the WC without help.

(c) Those who did not fall into either of these categories were classified as moderately dependent.
Table 2 shows the distribution of residents on the self-care scale.

Table 2 Self-care scale

\begin{tabular}{llr}
\hline & No. & $\%$ \\
\hline Severely dependent & 1589 & $22 \cdot 9$ \\
Moderately dependent & 2351 & $33 \cdot 8$ \\
Independent & 3007 & $43 \cdot 3$ \\
TOTAL & 6947 & $100 \cdot 0$ \\
\hline
\end{tabular}

\section{Continence scale}

This classified residents into one of four categories according to the following criteria:

(a) Residents who were incontinent of urine on average not less than once every 24 hours and who were also incontinent of faeces on average at least once every 24 hours were defined as doubly incontinent.

(b) Residents who were incontinent of urine on average not less than once every 24 hours, or who were incontinent of faeces on average at least once every 24 hours, were defined as incontinent.

(c) Residents who were incontinent of urine only or faeces only, or of both, but weekly or less often on average, were defined as mostly continent.

(d) Those who did not present any problems of incontinence were defined as fully continent.

Table 3 shows the distribution of residents on the continence scale.

Table 3 Continence scale

\begin{tabular}{lrr}
\hline & No. & \multicolumn{1}{c}{$\%$} \\
\hline Doubly incontinent & 463 & 6.7 \\
Incontinent & 955 & 13.7 \\
Mostly continent & 1102 & 15.9 \\
Fully continent & 4427 & $63 \cdot 7$ \\
TOTAL & 6947 & $100 \cdot 0$ \\
\hline
\end{tabular}

Social integration scale

This classified residents as socially aware and integrated, moderately well-integrated, or socially disengaged, on the basis of their awareness, behaviour, and sociability.

(a) Residents were classified as socially aware and integrated if they were normally fully aware of what wènt on around them, observed accepted social standards of behaviour in the home, and had formed stable friendships with other residents; or if, failing any one of these key criteria, they nevertheless did not display any of the characteristics incorporated in the definition of social disengagement given below.

(b) Residents were classified as socially disengaged if they were normally unaware of what went on around them, and behaved in such a way as to cause other residents actual distress or discomfort, and presented as solitary and isolated individuals; or if, failing any 
one of these key criteria, they nevertheless did not possess any of the characteristics incorporated in the definition of social integration given above.

(c) Those who did not fall into either of these two categories were classified as moderately wellintegrated.

Table 4 shows the distribution of residents on the social integration scale.

Table 4 Social integration scale

\begin{tabular}{lrr}
\hline & No. & \multicolumn{1}{c}{$\%$} \\
\hline Socially disengaged & 564 & $8 \cdot 1$ \\
Moderately well-integrated & 2969 & $42 \cdot 7$ \\
Socially aware and integrated & 3414 & $49 \cdot 1$ \\
TOTAL & 6947 & $100 \cdot 0$ \\
\hline
\end{tabular}

\section{Orientation scale}

This classified residents as mentally alert, slightly confused, and disorientated, using five variables-wandering, comprehension, memory, orientation, and awareness. Each variable comprised three values indicating absence of impairment, slight impairment, or more serious impairment.

(a) Residents were classified as mentally alert if they were coded as lacking any impairment on all five variables, or if they possessed nothing worse than a slight impairment on any one of them.

(b) Residents were classified as disorientated if they were coded as seriously impaired on at least four of the five variables, with any value on the fifth, or if they were coded as seriously impaired on any three variables and slightly impaired on both the others.

(c) Those who did not fall into either of these two categories were classified as slightly confused.

Table 5 shows the distribution of residents on the orientation scale.

Table 5 Orientation scale

\begin{tabular}{lrr}
\hline & No. & \multicolumn{1}{c}{$\%$} \\
\hline Disorientated & 670 & $9 \cdot 6$ \\
Slightly confused & 2646 & $38 \cdot 1$ \\
Mentally alert & 3631 & $52 \cdot 3$ \\
TOTAL & 6947 & $100 \cdot 0$ \\
\hline
\end{tabular}

Almost a quarter, or $1589(22.9 \%)$, of all residents were rated as severely dependent in terms of their capacity for self-care, and so were deemed to be heavily reliant on staff. A similar number and proportion (1418 and $20.4 \%$ ) of all residents were found to be incontinent. In general, it appears that residents were more handicapped in their physical functioning, such as mobility and capacity for personal care, than in their social and intellectual skills: only $564(8 \cdot 1 \%)$ were assessed as socially disengaged by virtue of their lack of awareness, social isolation, and unconventional behaviour; similarly, only $670(9.6 \%)$ were assessed as disorientated.

At the other extreme, the evidence shows that just over two in every five residents (3007, or $43.3 \%$ ) were able to get about the home without help and attend to their own personal needs. Almost two-thirds $(4427$, or $63.7 \%)$ were fully continent, $3414(49 \cdot 1 \%)$ were rated socially integrated, and $3631(52 \cdot 3 \%)$ were mentally alert.

\section{COMPARISON OF SCALES}

Largely as a result of the priority which has been given to the care of the elderly, many local authority social services departments have either commissioned studies or undertaken their own research on old people living in residential homes to provide information for use in planning services. The findings from a number of these studies have been reviewed by Hughes. and Wilkin. ${ }^{3}$ Sadly, there has been little collaborative effort in this field: each project tends to adopt a slightly different methodology, especially for the assessment of residents, and little attention is paid to problems of reliability and validity. Consequently, there are serious difficulties in trying to compare the evidence in the growing body of data.

As part of our overall research design, an attempt was made to compare the classifications and assessments of residents produced by our study with the ratings produced using other questionnaires developed for the same purpose. To this end, with the co-operation of officers in charge, the research schedules designed for use in five earlier dependency studies* were each applied in one of the homes from our study at approximately the time of the completion of the initial census. Space does not permit a full discussion of the results, which will be reported elsewhere, but the general findings are illuminating. Broadly, the assessment schedule and scales used in the present study seemed to correlate only weakly with the other scales, producing a slightly lower overall rating of dependency in the sample population than did either the Cleveland Functional Rating Scale or the NHS Operational Research Scale, and a significantly lower overall dependency rating than both Wright's Scale and the Clifton Assessment Procedure. Contrarily, the Devon schedule yielded a lower profile of dependency than the present study. A preliminary examination of the data and the questionnaires suggests two reasons for these results. In some cases there appears to be a measure of terminological confusion between the scales, which often use similar labels to denote rather different characteristics and types of behaviour. As a

-Details of these schedules are given in the Appendix. 
result, there is a temptation to expect a correlation where in fact the scales are measuring different aspects of a resident's functioning. More important, perhaps, the scales each seem to describe rather different parts of the dependency range. Our own scale in particular appears to be more discriminating at the top end of the dependency range where, our findings suggest, the span of abilities and disabilities is greater than these other scales allow. Our initial conclusion is that the underlying correlation between the scales is probably better than comparisons over their restricted and only partially overlapping ranges suggest, though further analysis of the data is currently under way.

\section{LEVELS OF DEPENDENCY}

The scales present a general picture of the pattern of abilities and infirmities in the census population; however, they still do not provide a full account of the extent and severity of dependency among individual residents. For this purpose, each resident was classified in terms of his or her combined rating on all four scales to produce a single rank ordering of overall levels of dependency.

This exercise involved making a number of assumptions about the relationship between the scales, notably about the relative weightings to be assigned to each of them. For analytical purposes, each category on any one of the scales was given the same weighting as the equivalent category on the other scales. On the continence scale, it was decided to subsume both doubly incontinent and incontinent residents into one category representing severe incontinence. This produced four scales each divided into three supposedly equivalent categories measuring high $(\mathrm{H})$, medium $(\mathrm{M})$, and low (L) dependency. For the purpose of constructing a single

Table 6 Levels of dependency among residents of homes for the elderly

\begin{tabular}{|c|c|c|c|c|}
\hline Dependency rating & Scale profile & No. & $\%$ & \\
\hline High & $\begin{array}{l}\text { HHHH } \\
\text { HHHM } \\
\text { HHHL } \\
\text { HHMM } \\
\text { HHML }\end{array}$ & $\begin{array}{r}201 \\
248 \\
25 \\
455 \\
156\end{array}$ & $\begin{array}{l}2.9 \\
3.6 \\
0.4 \\
6 \cdot 5 \\
2.2\end{array}$ & $15 \cdot 6$ \\
\hline Medium & $\begin{array}{l}\text { HMMM } \\
\text { HHLL } \\
\text { HMML } \\
\text { MMMM } \\
\text { HMLL }\end{array}$ & $\begin{array}{r}350 \\
81 \\
376 \\
229 \\
300\end{array}$ & $\begin{array}{l}5 \cdot 0 \\
1 \cdot 2 \\
5 \cdot 4 \\
3 \cdot 3 \\
4 \cdot 3\end{array}$ & $19 \cdot 2$ \\
\hline$\left.\right|_{\text {TOTAL }}$ & $\begin{array}{l}\text { MMML } \\
\text { HLLL } \\
\text { MMLL } \\
\text { MLLL } \\
\text { LLLL }\end{array}$ & $\begin{array}{r}583 \\
208 \\
882 \\
1223 \\
1630 \\
6947\end{array}$ & $\begin{array}{r}8 \cdot 4 \\
3 \cdot 0 \\
12 \cdot 7 \\
17 \cdot 6 \\
23 \cdot 5 \\
100 \cdot 0\end{array}$ & $\begin{array}{r}65 \cdot 2 \\
100 \cdot 0\end{array}$ \\
\hline
\end{tabular}

dependency rating, residents were classified according to the number of high $(\mathrm{H})$, medium (M), and low $(L)$ rankings they accumulated, irrespective of the pattern of their rankings on the individual scales. Thus, a resident rated HHLL on the four scales would be classed with others rated HLHL, HLLH, LHHL, LLHH. The results are presented in Table 6.

Of the total of 6947 residents, $3735(53 \cdot 8 \%)$ were ranked on one of the lowest three levels of dependency. At the other extreme, 474 (6.9\%) of residents were rated as highly dependent on at least three of the scales, and $929(13.4 \%)$ of the census population was classified in the four most severe categories of dependency.

\section{Conclusion}

Two qualifications should be kept in mind about the findings of the census reported here. Firstly, the census was designed to assess the functioning of residents in the specific context of the old people's homes in which they lived. It could be misleading to draw conclusions from these data alone about how the residents might manage, or might have managed, in other environments offering a different level of care and support or a different type of medical treatment. Secondly, the simple data alone provide no insight into the ways in which dependency is moderated or created, and especially into the part played in this process by the quality of the residential setting and the institutional way of life as against the natural effects of ageing, illness, and disease-although further research on this issue is planned.

These points aside, however, the census clearly demonstrates the present extent of dependency among residents of homes for the elderly, since $2.9 \%$ of the enumerated population were assessed as highly dependent on all four scales. This group is likely to present very pressing problems of management and control. At the same time, a further $13.9 \%$ of residents, rated as highly dependent on at least two of the scales, may be identified as substantially reliant on the care and support of residential staff.

On the other hand, as the census shows, almost a quarter of the residents were competent in their functioning on all four scales, and more than half $(53.8 \%)$ were clustered onto the bottom three rankings of the scale profiles (Table 6).

The implication is that the burden of care on residential staffs derives largely from the demands made on their time and skills by a minority of severely dependent residents. Indeed, the evidence strongly suggests that local authority homes for the elderly are 
trying to cope simultaneously with two conflicting tasks: on the one hand, providing comprehensive personal and often nursing care to physically and mentally infirm old people, and, on the other hand, creating an environment for living which safeguards the dignity and personal freedom of the more independent residents.

We acknowledge the financial support for this project received from the University of Sheffield Research Fund.

Reprints from Mr. T. Booth, Joint Unit for Social Services Research, Department of Sociological Studies, University of Sheffield, Sheffield S10 2TN.

\section{References}

${ }^{1}$ Booth T. Forging ahead. Community Care 1980; 332: 24-6.

${ }^{1 a}$ Booth $\mathrm{T}$, et al. A reliable assessment schedule for use in homes for the elderly. Social Work Service 1982; 29.

${ }^{2}$ Booth T. Measuring dependency. Community Care 1980; 300: 15-8.

${ }^{3}$ Hughes B, Wilkin D. Residential care of the elderly: $a$ review of the literature. Manchester: Department of Community Medicine, University of Manchester, 1980.
Appendix

The research schedules used were those developed for the following studies:

(i) Residential accommodation for the elderly 1978-79. Research and Training Section, Devon County Council Social Services Department, August 1979.

(ii) Schiphorst B. Development of a functional rating chart for the elderly: application in Cleveland and Sunderland social services departments. Social Work Service 1979; 19: 3-10.

(iii) Pattie AH, Gilleard CJ. Manual for the Clifton assessment procedure for the elderly (CAPE). Sevenoaks: Hodder and Stoughton, 1979.

Gilleard CJ, Pattie AH, Dearman G. Behavioural disabilities in psychogeriatric patients and residents of old people's homes. J Epidemiol Community Health 1980; 34: 106-10.

(iv) Wright KG, Cairns JA, Snell MC. The costs of alternative patterns of care for the elderly. York: Institute of Social and Economic Research, University of York, 1981.

(v) Vaswani N, Parker C, Mitchell K. OR study of care of the elderly in Calderdale. Report of the OR research service of the Department of Health and Social Security. ORS note 41/77. London: DHSS, 1978. 